DOI: $10.17516 / 1997-1397-2020-13-1-87-96$

УДК 517.55

\title{
Hypergeometric Series and the Mellin-Barnes Integrals for Zeros of a System of Laurent Polynomials
}

Vladimir R. Kulikov*

Siberian Federal University

Krasnoyarsk, Russian Federation

Received 13.09.2019, received in revised form 06.11.2019, accepted 20.12.2019

\begin{abstract}
In the article we present a criterion for convergence of the Mellin-Barnes integral for zeros of a system of Laurent polynomials. Also we give a hypergeometric series for these zeros.
\end{abstract}

Keywords: Mellin-Barnes integrals, hypergeometric series, Laurent polynomials.

Citation: V.R.Kulikov, Hypergeometric Series and the Mellin-Barnes Integrals for Zeros of a System of Laurent Polynomials, J. Sib. Fed. Univ. Math. Phys., 2020, 13(1), 87-96.

DOI: $10.17516 / 1997-1397-2020-13-1-87-96$.

\section{Introduction}

In $1921 \mathrm{Hj}$. Mellin wrote down an integral representing a solution $y(x)$ of a reduced algebraic equation of the form

$$
y^{n}+x_{1} y^{n-1}+\ldots+x_{n-1} y-1=0 .
$$

This integral has a non-empty domain of convergence, it is defined by conditions on arguments $\theta_{j}=\arg x_{j}$. A complete description of the convergence domain has been obtained relatively recently in the paper by I. A. Antipova [3].

In the present paper we study the same problem in several variables. Consider a system of algebraic equations of the form

$$
\mathbf{y}^{\omega^{(j)}}+\sum_{\lambda \in \Lambda^{(j)}} x_{\lambda}^{(j)} \mathbf{y}^{\lambda}-1=0, j=1, \ldots, n,
$$

where $\Lambda^{(j)} \subset \mathbb{Z}^{n}$, and $\omega^{(j)}$ is a column vector, the matrix made of columns $\omega^{(j)}$ we denote by $\Omega$. Let us also introduce the notation $\Lambda:=\bigsqcup_{j=1}^{n} \Lambda^{(j)}$ for a disjunctive union of sets $\Lambda^{(j)}$, the cardinality of $\Lambda$ we denote by $N$. By $\overline{\Lambda^{(j)}}$ we shall denote the set $\Lambda^{(j)} \cup\left\{\omega^{(j)}\right\}$, analogously $\bar{\Lambda}=\bigsqcup_{j=1}^{n} \overline{\Lambda^{(j)}}$.

The set of coefficients of the system (1) runs over the vector space $\mathbb{C}^{\lambda} \cong \mathbb{C}_{\mathbf{x}}^{N}$, where coordinates of points $\mathbf{x}=\left(x_{\lambda}\right)$ are indexed by the elements $\lambda \in \Lambda$. A group of coordinates corresponding to indices $\lambda \in \Lambda^{(i)}$ we, as a rule, write as $x_{\lambda}^{(i)}$, having identified $\mathbb{C}^{\Lambda}$ with $\mathbb{C}^{\Lambda^{(1)}} \times \ldots \times \mathbb{C}^{\Lambda^{(n)}}$; sometimes for elements of $\mathbb{C}^{\Lambda^{(i)}}$ we use the notation $x_{\lambda}, \lambda \in \Lambda^{(i)}$. Denote also by $X$ the diagonal matrix with $x_{\lambda}$ on the diagonal $(X=\operatorname{diag}[\mathbf{x}])$.

\footnotetext{
*v.r.kulikov@mail.ru https://orcid.org/0000-0002-2291-0449 (C) Siberian Federal University. All rights reserved
} 
The set $\Lambda$ we will also treat as a matrix

$$
\Lambda=\left(\Lambda^{(1)}, \ldots, \Lambda^{(n)}\right)=\left(\lambda^{1}, \ldots, \lambda^{N}\right)
$$

whose columns are the vectors $\lambda^{k}=\left(\lambda_{1}^{k}, \ldots, \lambda_{n}^{k}\right)$ of exponents of monomials of the system (1). Here we mean that a block $\Lambda^{(i)}$ of the matrix $\Lambda$ corresponds to the $i$ th equation of the system (1); enumeration of columns $\lambda^{k}$ in each block $\Lambda^{(i)}$ is arbitrary but fixed.

Denote by $\chi$ the characteristic matrix of the set $\Lambda$.

In this notation the system (1) can be written in a matrix form:

$$
\mathbf{y}^{\Omega}+\mathbf{y}^{\Lambda} X \chi^{T}-I=0
$$

We are interested in a branch of a solution $\mathbf{y}(\mathbf{x})=\left(y_{1}(\mathbf{x}), \ldots, y_{n}(\mathbf{x})\right)$ of the system (1) with the condition $\mathbf{y}(\mathbf{0})=(1, \ldots, 1)$, which we call the principal solution. Following [2,4], to a monomial $\mathbf{y}^{\mu}=y_{1}^{\mu_{1}} \ldots y_{n}^{\mu_{n}}$ of the principal solution $\mathbf{y}=\mathbf{y}(\mathbf{x})$ of the system we put into the correspondence the Mellin-Barnes integral:

$$
\mathbf{y}^{\mu}(\mathbf{x}) \rightarrow \frac{1}{(2 \pi i)^{N}} \int_{\gamma+i \mathbb{R}^{N}} \frac{\Gamma(\mathbf{u}) \Gamma\left(\Omega^{-1} \mu-\Omega^{-1} \Lambda \mathbf{u}\right)}{\Gamma\left(\Omega^{-1} \mu-\Omega^{-1} \Lambda \mathbf{u}+\chi \mathbf{u}+I\right)} Q(\mathbf{u}) \mathbf{x}^{-\mathbf{u}} d u
$$

where the vector $\gamma$ is from the polyhedron

$$
\left\{\mathbf{u} \in \mathbb{R}_{>0}^{N}:\left\langle\varphi_{j}, \mathbf{u}\right\rangle<\mu_{j}, j=1, \ldots, n\right\}
$$

and $Q(\mathbf{u})$ is a polynomial given by the determinant

$$
Q(\mathbf{u})=\operatorname{det}\left(\operatorname{diag}\left[\Omega^{-1} \cdot(\mu-\Lambda \cdot \mathbf{u})\right]+\Omega^{-1} \cdot \Lambda \cdot \operatorname{diag}[\mathbf{u}] \chi^{T}\right)
$$

The integral (3) is obtained by a formal computation of the Mellin transform of $\mathbf{y}^{\mu}(\mathbf{x})$ using linearization.

Consider the following matrices made of exponents of monomials of the system (1):

$$
\left(\begin{array}{ccc}
\lambda_{1}^{(1)} & \ldots & \lambda_{1}^{(n)} \\
\vdots & \ddots & \vdots \\
\lambda_{n}^{(1)} & \cdots & \lambda_{n}^{(n)}
\end{array}\right)
$$

where each column vector $\lambda^{(j)}=\left(\lambda_{1}^{(j)} \ldots \lambda_{n}^{(j)}\right)^{T}$ runs over the corresponding set $\overline{\Lambda^{(j)}}$.

Theorem 1. The integral (3) corresponding to a system of algebraic equations (1) has a nonempty domain of convergence and represents the monomial function of the solution if and only if the determinants of all matrices of the form (5) are non-zero and have the same sign.

Note that in [9] and [10] analogous results have been obtained for systems with a diagonal matrix $\omega$ and non-negative exponents of monomials $\lambda \in \Lambda^{(j)}$. Applications of these results for study of discriminants of systems are given in [11]. 


\section{Convergence of the Mellin-Barnes integral}

In this section we prove the convergence of the Mellin-Barnes integral (3) under hypothesis of Theorem 1.

Recall that a multiple Mellin-Barnes integral has the form

$$
\Phi(\mathbf{z})=\frac{1}{(2 \pi i)^{m}} \int_{\gamma+i \mathbb{R}^{m}} \frac{\Gamma(A \cdot \mathbf{s}+\mathbf{c})}{\Gamma(B \cdot \mathbf{s}+\mathbf{d})} \mathbf{z}^{-\mathbf{s}} d s,
$$

where $A \in \mathbb{R}^{m \times p}, B \in \mathbb{R}^{m \times q}, \mathbf{c} \in \mathbb{C}^{p}, \mathbf{d} \in \mathbb{C}^{q}, \mathbf{z}, \mathbf{s} \in \mathbb{C}^{m}$, and the vector $\gamma$ is chosen such that the integration set $\gamma+i \mathbb{R}^{m}$ does not contain poles of $\Gamma$ functions of the numerator.

We shall assume that the variable $z$ varies in a Riemannian covering of the complex algebraic torus $(\mathbb{C} \backslash\{0\})^{m}$, consequently, the factors in the integral kernel are defined as

$$
z_{j}^{-s_{j}}=e^{-s_{j} \log z_{j}}, \arg z_{j} \in \mathbb{R} .
$$

Denote $\theta=\operatorname{Arg} z=\left(\arg z_{1}, \ldots, \arg z_{m}\right)$ and introduce the function

$$
g(\mathbf{v})=\sum_{j=1}^{p}\left|\left\langle A_{j}, \mathbf{v}\right\rangle\right|-\sum_{k=1}^{q}\left|\left\langle B_{k}, \mathbf{v}\right\rangle\right|,
$$

where $A_{j}$ and $B_{k}$ are rows of matrices $A$ and $B$, respectively.

The next theorem gives a description of the convergence domain of a multiple Mellin-Barnes integral.

Theorem 2 (Nilsson, Passare, Tsikh). For an integration set $\gamma+i \mathbb{R}^{m}$ that does not contain singularities of the integrand the convergence domain of the Mellin-Barnes integral (6) has the form $\operatorname{Arg}^{-1}(U)$, where

$$
U=\bigcap_{\|v\|=1}\left\{\theta \in \mathbb{R}^{m}:|\langle\mathbf{v}, \theta\rangle|<\frac{\pi}{2} g(\mathbf{v})\right\} .
$$

In the case when the set $U$ is not empty it coincides with the interior $\Theta^{\circ}$ of the polyhedron

$$
\Theta=\left\{\theta \in \mathbb{R}^{m}:\left|\left\langle\mathbf{v}_{\nu}, \theta\right\rangle\right| \leqslant \frac{\pi}{2} g\left(\mathbf{v}_{\nu}\right), \nu=1, \ldots, d\right\},
$$

where $\pm \mathbf{v}_{1}, \ldots, \pm \mathbf{v}_{d}$ is the set of unit vectors generating the fan $K$ defined by a decomposition of $\mathbb{R}^{m}$ by hyperplanes $\left\langle A_{j}, \mathbf{v}\right\rangle=0, j=1, \ldots, p$ and $\left\langle B_{k}, \mathbf{v}\right\rangle=0, k=1, \ldots, q$.

Thus, the convergence domain of the integral (6) is not empty if the function $g(v)$ is positive on the compact set (sphere) $\|\mathbf{v}\|=1$. Since $g(\mathbf{v})$ is homogeneous, this is equivalent to its positivity for $\mathbf{v} \neq 0$.

As has been established in earlier papers, the convergence domain of a Mellin-Barnes integral does not depend on the presence of a polynomial factor $Q(u)$.

For the integral (3) the function $g(v)$ is

$$
g(v)=\|\mathbf{v}\|+\left\|\Omega^{-1} \Lambda \mathbf{v}\right\|-\left\|\left(\chi-\Omega^{-1} \Lambda\right) \mathbf{v}\right\|
$$

where $\|\mathbf{v}\|=\left|v_{1}\right|+\ldots+\left|v_{N}\right|$.

The matrix $\Phi=\Omega^{-1} \Lambda$ inherits its block structure from the matrix $\Lambda$ compatible with the characteristic matrix $\chi$; the blocks of this matrix are denoted by $\Phi^{(j)}$. In [9] it has been shown that in this case the function $g(\mathbf{v})$ vanishes only for $v=0$ if and only if all diagonal minors 
of matrices $\varphi=\left(\varphi^{(1)}, \ldots, \varphi^{(n)}\right)$ are positive, here $\varphi^{(j)}$ is an arbitrary column vector of the matrix $\Phi^{(j)}$.

Consider a diagonal minor of order $p$ of this matrix, it may be obtained as the determinant of a product of two rectangular matrices:

$$
\varphi_{j_{1}, \ldots, j_{p}}^{j_{1}, \ldots, j_{p}}=\operatorname{det}\left(\left(\Omega^{-1}\right)_{j_{1}, \ldots, j_{p}} \cdot \lambda^{j_{1}, \ldots, j_{p}}\right),
$$

where $\lambda=\Omega \varphi$.

By the Cauchy-Binet formula, the determinant of a product of two such matrices is a sum of products of minors of these matrices:

$$
\varphi_{j_{1}, \ldots, j_{p}}^{j_{1}, \ldots, j_{p}}=\sum_{1 \leqslant k_{1}<\ldots<k_{p} \leqslant n}\left(\Omega^{-1}\right)_{j_{1}, \ldots, j_{p}}^{k_{1}, \ldots, k_{p}} \cdot \lambda_{k_{1} \ldots, k_{p}}^{j_{1}, \ldots, j_{p}}
$$

By definition,

$$
\Omega^{-1}=\frac{\operatorname{adj} \Omega^{T}}{|\Omega|}
$$

Therefore, by the Jacobi identity the minor $\left(\Omega^{-1}\right)_{j_{1}, \ldots, j_{p}}^{k_{1} \ldots, k_{p}}$ can be computed as:

$$
\left(\Omega^{-1}\right)_{j_{1}, \ldots, j_{p}}^{k_{1} \ldots, k_{p}}=\frac{(-1)^{\sigma}}{|\Omega|} \Omega_{k_{p+1} \ldots, k_{n}}^{j_{p+1}, \ldots, j_{n}},
$$

where $\sigma$ is the order of the permutation

$$
\left(\begin{array}{lll}
j_{1} & \cdots & j_{n} \\
k_{1} & \ldots & k_{n}
\end{array}\right)
$$

Substituting these expressions into the formula for the minor $\varphi_{j_{1}, \ldots, j_{p}}^{j_{1}, \ldots, j_{p}}$ and using the Laplace expansion along several columns we get

$$
\varphi_{j_{1}, \ldots, j_{p}}^{j_{1}, \ldots, j_{p}}=\frac{|A|}{|\Omega|}>0
$$

where $A$ is the matrix whose columns with numbers $j_{s}$ are equal to $\lambda^{\left(j_{s}\right)}$, and all the remaining columns are the corresponding columns of $\Omega$, i.e. $A$ is a matrix of the form (5).

Since the choice of a matrix $\varphi$ and an order of a minor $p$ is arbitrary, it follows that all determinants of matrices of the form (5) has the same sign. Thus, we have proved that under the hypothesis of Theorem 1 the Mellin-Barnes integral corresponding to a solution of the system (1) converges.

\section{Solution of the system as a Taylor series}

Consider the system (2):

$$
\mathbf{y}^{\Omega}+\mathbf{y}^{\Lambda} X \chi^{T}-I=0
$$

The following statement holds

Theorem 3. The monomial $\widehat{\mathbf{y}}^{\mu}(\mathbf{x})$ of the principal solution of the system (2) is given by the Taylor series

$$
\widehat{\mathbf{y}}^{l}(\mathbf{x})=\sum_{\mathbf{k} \in \mathbb{Z}_{\geqslant}^{N}} c_{\mathbf{k}} \mathbf{x}^{\mathbf{k}}
$$


with the coefficients

$$
c_{k}=\frac{(-1)^{|\mathbf{k}|}}{\mathbf{k} !} \frac{\Gamma\left(\Omega^{-1} \cdot \mu+\Omega^{-1} \cdot \Lambda \cdot \mathbf{k}\right)}{\Gamma\left(\Omega^{-1} \cdot \mu+\Omega^{-1} \cdot \Lambda \cdot \mathbf{k}-\chi \cdot \mathbf{k}+I\right)} \cdot Q(\mathbf{k}),
$$

where $Q(\mathbf{k})=\operatorname{det}\left(\operatorname{diag}\left[\Omega^{-1} \cdot(\mu+\Lambda \cdot \mathbf{k})\right]-\Omega^{-1} \cdot \Lambda \cdot \operatorname{diag}[\mathbf{k}] \cdot \chi^{T}\right)$.

Proof. First, let us linearize the system (2). Consider it as a system in $\mathbb{C}_{\mathbf{x}}^{N} \times \mathbb{C}_{\mathbf{y}}^{n}$ and make in $\mathbb{C}^{N+n}$ the following change of variables:

$$
y=W^{-\Omega^{-1}} ; x=\xi \odot W^{\Omega^{-1} \Lambda-\chi} .
$$

In the new variable the system becomes

$$
W=\xi \chi^{T}+I .
$$

Represent the inverse $\xi(\mathbf{x})$ as an implicit function given by the system of equations

$$
F(\xi, \mathbf{x})=\xi \odot W^{\Omega^{-1} \Lambda-\chi}-\mathbf{x} .
$$

Zeroes of these functions define the change of linearization. Therefore, the monomial function of the solution can be found by A. P. Yuzhakov's logarithmic residue formula. According to this formula

$$
y^{\mu}(\mathbf{x})=\frac{1}{(2 \pi i)^{N}} \int_{\Gamma_{\varepsilon}} \frac{y^{\mu}(\xi) \Delta(\xi) d \xi}{F^{I}(\xi, \mathbf{x})}
$$

where $\Gamma_{\varepsilon}=\left\{\xi \in \mathbb{C}^{N}:\left|\xi_{\lambda}\right|=\varepsilon, \lambda \in \Lambda\right\}, \Delta(\xi)$ is the Jacobian of the mapping $F(\xi, x)$ with respect to variables $\xi$ (notice that the Jacobian does not contain variables $x$ ), $F^{I}(\xi, x)$ denotes the product $F_{1}(\xi, \mathbf{x}) \cdot \ldots \cdot F_{N}(\xi, \mathbf{x})$. The radius $\varepsilon$ is chosen in such a way that the corresponding polydisc lies outside zeroes of the Jacobian $\Delta(\xi)$.

Lemma 1. The Jacobian of $F(\xi, \mathbf{x})$ with respect to $\xi$ is

$$
\Delta(\xi)=W^{\left(\Omega^{-1} \Lambda-\chi\right) I-I} \operatorname{det}\left(E+\Omega^{-1} \Lambda \cdot \Xi \cdot \chi^{T}\right),
$$

here and further on $\Xi=\operatorname{diag}[\xi]$.

Proof. The component of the mapping $F(\xi, \mathbf{x})$ with the index $\lambda^{(j)} \in \Lambda^{(j)}, j=1, \ldots, n$ has the form

$$
F_{\lambda^{(j)}}=\xi_{\lambda^{(j)}} \prod_{k=1}^{n} W_{k}^{\left(\Omega^{-1} \Lambda-\chi\right)_{k}^{(j)}}
$$

here $\left(\Omega^{-1} \Lambda-\chi\right)_{k}^{\lambda^{(j)}}$ denotes the $k$-th component of the column with the index $\lambda^{(j)}$ of the matrix $\Omega^{-1} \Lambda-\chi$.

Non-diagonal elements of the Jacobian are

$$
\frac{\partial F_{\lambda^{(j)}}}{\partial \xi_{\eta^{(i)}}}=\xi_{\lambda^{(j)}}\left(\Omega^{-1} \Lambda-\chi\right)_{i}^{\lambda^{(j)}} \prod_{k=1}^{n} W_{k}^{\left(\Omega^{-1} \Lambda-\chi\right)_{k}^{\lambda^{(j)}}-\delta_{k}^{i}}, \quad \lambda^{(j)} \in \Lambda^{(j)}, \quad \eta^{(i)} \in \Lambda^{(i)},
$$

and if $i=j$ then $\lambda^{(j)} \neq \eta^{(i)}$. 
Its diagonal elements has the form

$$
\frac{\partial F_{\lambda^{(j)}}}{\partial \xi_{\lambda^{(j)}}}=\prod_{k=1}^{n} W_{k}^{\left(\Omega^{-1} \Lambda-\chi\right)_{k}^{\lambda^{(j)}}}\left(1+\xi_{\lambda^{(j)}}\left(\Omega^{-1} \Lambda-\chi\right)_{j}^{\lambda^{(j)}} W_{j}^{-1}\right), \quad \lambda^{(j)} \in \Lambda^{(j)},
$$

where $\delta_{k}^{j}$ is the Kronecker delta.

From each row of the Jacobian we factor out

$$
W^{\left(\Omega^{-1} \Lambda-2 \chi\right)^{\lambda(j)}}=\prod_{k=1}^{n} W_{k}^{\left(\Omega^{-1} \Lambda-\chi\right)_{k}^{\lambda^{(j)}}-\delta_{k}^{j}} .
$$

Then, before the Jacobian we have the factor $W^{\left(\Omega^{-1} \Lambda-2 \chi\right) I}$, and the elements of the Jacobian become

$$
\frac{\partial F_{\lambda^{(j)}}}{\partial \xi_{\eta^{(i)}}}=\xi_{\lambda^{(j)}}\left(\Omega^{-1} \Lambda-\chi\right)_{i}^{\lambda^{(j)}}, \quad \lambda^{(j)} \in \Lambda^{(j)}, \quad \eta^{(i)} \in \Lambda^{(i)},
$$

outside the diagonal, and

$$
\frac{\partial F_{\lambda^{(j)}}}{\partial \xi_{\lambda^{(j)}}}=W_{j}+\xi_{\lambda^{(j)}}\left(\Omega^{-1} \Lambda-\chi\right)_{j}^{\lambda^{(j)}}, \quad \lambda^{(j)} \in \Lambda^{(j)}
$$

on the diagonal.

In each $i$-th block-column of the obtained determinant subtract one column of this block from all other columns of the block, the chosen columns we shall call marked, and their indices are denoted by $\eta^{(i)}$, while $\Lambda^{(i)}:=\Lambda(i) \backslash\left\{\eta^{(i)}\right\}$ denote indices of not marked columns of the $i$ th block.

The elements of the Jacobian then take the form:

$$
\frac{\partial F_{\lambda^{(j)}}}{\partial \xi_{\eta^{(i)}}}=0 ; \lambda^{(j)} \in \Lambda^{(j)}, \quad \eta^{(i)} \in{ }^{\prime} \Lambda^{(i)}, i \neq j,
$$

for those in non-diagonal blocks in not marked columns.

$$
\frac{\partial F_{\eta^{(j)}}}{\partial \xi_{\eta^{(j)}}}=-W_{j}, \quad \eta^{(j)} \in \Lambda^{\prime} \Lambda^{(j)},
$$

for elements in diagonal blocks in marked columns.

$$
\frac{\partial F_{\lambda^{(j)}}}{\partial \xi_{\lambda^{(j)}}}=W_{j}, \quad \lambda^{(j)} \in \Lambda^{\prime} \Lambda^{(j)}
$$

for diagonal elements in not marked columns.

$$
\frac{\partial F_{\eta^{(j)}}}{\partial \xi_{\eta^{(j)}}}=W_{j}+\xi_{\eta^{(j)}}\left(\Omega^{-1} \Lambda-\chi\right)_{j}^{\eta^{(j)}}
$$

for diagonal elements in marked columns.

$$
\frac{\partial F_{\lambda}}{\partial \xi_{\eta^{(i)}}}=\xi_{\lambda}\left(\Omega^{-1} \Lambda-\chi\right)_{i}^{\lambda}, \quad \lambda \in \Lambda \backslash\left\{\eta^{(i)}\right\},
$$

for all other elements in marked columns, and

$$
\frac{\partial F_{\lambda^{(j)}}}{\partial \xi_{\eta^{(j)}}}=0 ; \quad \lambda^{(j)}, \eta^{(j)} \in{ }^{\prime} \Lambda^{(j)}, \lambda^{(j)} \neq \eta^{(j)},
$$


for all remaining elements.

Now in each $j$ th block-row add to the row with the index $\eta^{(j)}$ all other rows of this block to get

$$
\begin{gathered}
\frac{\partial F_{\eta^{(j)}}}{\partial \xi_{\eta^{(i)}}}=W_{j} \delta_{i}^{j}+\sum_{\lambda^{(j)} \in \Lambda^{(j)}} \xi_{\lambda^{(j)}}\left(\Omega^{-1} \Lambda-\chi\right)_{i}^{\lambda^{(j)}}, \\
\frac{\partial F_{\eta^{(j)}}}{\partial \xi_{\eta^{(j)}}}=0, \quad \eta^{(j)} \in \in^{\prime} \Lambda^{(j)} .
\end{gathered}
$$

Since non-zero elements are now only in marked columns and on the principal diagonal, the Jacobian can be reduced to a determinant of order $n$ :

$$
\frac{\partial F}{\partial \xi}=W^{\left(\Omega^{-1} \Lambda-\chi\right) I-I} \operatorname{det}\left(E+\Omega^{-1} \Lambda \cdot \Xi \cdot \chi^{T}\right) .
$$

This proves the lemma.

Substitute now the expression for the Jacobian as well as the expression for $\mathbf{y}^{\mu}(\xi)$ into (11)

$$
\mathbf{y}^{\mu}(\mathbf{x})=\frac{1}{(2 \pi i)^{N}} \int_{\Gamma_{\varepsilon}} \frac{W^{-\Omega^{-1} \mu} \cdot W^{\left(\Omega^{-1} \Lambda-\chi\right) I-I} \operatorname{det}\left(E+\Omega^{-1} \Lambda \cdot \Xi \cdot \chi^{T}\right)}{\left(\xi \odot W^{\left(\Omega^{-1} \Lambda-\chi\right)}-\mathbf{x}\right)^{I}} d \xi
$$

and reduce the fraction in the integrand

$$
\mathbf{y}^{\mu}(\mathbf{x})=\frac{1}{(2 \pi i)^{N}} \int_{\Gamma_{\varepsilon}} \frac{W^{-\Omega^{-1} \mu-I} \operatorname{det}\left(E+\Omega^{-1} \Lambda \cdot \Xi \cdot \chi^{T}\right)}{\xi^{I}\left(I-\mathbf{x} \odot \xi^{-E} \odot W^{\chi^{-\Omega^{-1}} \Lambda}\right)^{I}} d \xi .
$$

There exists $\delta$ such that for any $\xi \in \Gamma_{\varepsilon}$ and $\|\mathbf{x}\|<\delta$ we have the inequality $\mathbf{x} \odot \xi^{-E} \odot$ $W^{\chi-\Omega^{-1} \Lambda}<I$, therefore we can represent the integrand as a geometric series

$$
\begin{aligned}
\mathbf{y}^{\mu}(\mathbf{x}) & =\frac{1}{(2 \pi i)^{N}} \int_{\Gamma_{\varepsilon}} \frac{W^{-\Omega^{-1} \mu-I} \operatorname{det}\left(E+\Omega^{-1} \Lambda \cdot \Xi \cdot \chi^{T}\right)}{\xi^{I}} \times \\
& \times\left(\sum_{\mathbf{k} \in \mathbb{Z}_{\geqslant}^{N}} \mathbf{x}^{\mathbf{k}} \cdot \xi^{-\mathbf{k}} \cdot W^{\left(\chi-\Omega^{-1} \Lambda\right) \mathbf{k}}\right) d \xi .
\end{aligned}
$$

Now change the order of integration in (15):

$$
\mathbf{y}^{\mu}(\mathbf{x})=\sum_{\mathbf{k} \in \mathbb{Z}_{\geqslant}^{N}} c_{\mathbf{k}} \mathbf{x}^{\mathbf{k}}
$$

here the coefficients $c_{\mathbf{k}}$ are given by

$$
c_{\mathbf{k}}=\frac{1}{(2 \pi i)^{N}} \int_{\Gamma_{\varepsilon}} \frac{W^{-\Omega^{-1}(\mu+\Lambda \mathbf{k})+\chi \mathbf{k}-I}}{\xi^{\mathbf{k}+I}} \operatorname{det}\left(E+\Omega^{-1} \Lambda \cdot \Xi \cdot \chi^{T}\right) d \xi .
$$

The coefficients of the obtained series can be computed by the Cauchy formula

$$
c_{\mathbf{k}}=\left.\frac{1}{\mathbf{k} !} \frac{\partial^{\mathbf{k}}}{\partial \xi^{\mathbf{k}}}\left(W^{-\Omega^{-1}(\mu+\Lambda \mathbf{k})+\chi \mathbf{k}-I} \operatorname{det}\left(E+\Omega^{-1} \Lambda \cdot \Xi \cdot \chi^{T}\right)\right)\right|_{\xi=0} .
$$

The computation of the derivatives gives

$$
c_{\mathbf{k}}=\frac{(-1)^{\mathbf{k}}}{\mathbf{k} !} \frac{\Gamma\left(\Omega^{-1}(\mu+\Lambda \cdot \mathbf{k})\right)}{\Gamma\left(\Omega^{-1}(\mu+\Lambda \cdot \mathbf{k})-\chi \cdot k+I\right)} Q(k),
$$

where $Q(\mathbf{k})=\operatorname{det}\left(\operatorname{diag}\left[\Omega^{-1}(\mu+\Lambda \mathbf{k})\right]-\Omega^{-1} \Lambda \cdot \operatorname{diag}[\mathbf{k}] \cdot \chi^{T}\right)$. 


\section{Computation of the Mellin-Barnes integral}

In this section we show that a convergent integral corresponding to a solution of a system of algebraic equations can be computed as a hypergeometric series, which coincides with a hypergeometric series representing solution of this system.

Consider a system of two trinomials

$$
\begin{aligned}
& y_{1}^{4}+x_{1} y_{1}^{2} y_{2}^{-1}-1=0 \\
& y_{2}^{4}+x_{2} y_{1}^{-1} y_{2}^{2}-1=0 .
\end{aligned}
$$

It is easy to check that this system satisfies the condition for the convergence of the MellinBarnes integral, which for the monomial of the solution $y^{\mu}(x)$ has the form

$$
\frac{1}{(2 \pi i)^{2}} \int_{\gamma+i \mathbb{R}^{2}} \frac{\Gamma\left(u_{1}\right) \Gamma\left(u_{2}\right) \Gamma\left(\frac{1}{4}\left(\mu_{1}-2 u_{1}+u_{2}\right)\right) \Gamma\left(\frac{1}{4}\left(\mu_{2}+u_{1}-2 u_{2}\right)\right)}{\Gamma\left(\frac{1}{4}\left(\mu_{1}+2 u_{1}+u_{2}\right)+1\right) \Gamma\left(\frac{1}{4}\left(\mu_{2}+u_{1}+2 u_{2}\right)+1\right)} Q(u) x^{-u} d u,
$$

where

$$
Q(u)=\frac{1}{16}\left(\mu_{1} \mu_{2}+\mu_{1} u_{1}+\mu_{2} u_{2}\right) .
$$

To compute the integral we use the principle of separating cycles of A. K. Tsikh. This principle applies for computation of integrals

$$
\frac{1}{(2 \pi i)^{s}} \int_{\Delta_{g}} \frac{h(\mathbf{z}) d \mathbf{z}}{f_{1}(\mathbf{z}) \ldots f_{s}(\mathbf{z})}
$$

of the Grothendieck type where poles of the meromorphic integrand are associated to a proper holomorphic mapping $\mathbf{f}=\left(f_{1}, \ldots, f_{s}\right): \mathbb{C}^{s} \rightarrow \mathbb{C}^{s}$, and the integration set $\Delta_{g}$ is the distinguished boundary of the polyhedron $\Pi_{\mathrm{g}}$ associated to another proper holomorphic mapping $\mathbf{g}=\left(g_{1}, \ldots, g_{s}\right): \mathbb{C}^{s} \rightarrow \mathbb{C}^{s}$. When the mappings $\mathbf{f}$ and $\mathbf{g}$ coincide, the integral (17) is equal to a sum of Grothendieck residues of the integrand over all zeroes of $\mathbf{f}$ in $\Pi_{g}$. Indeed, in this case the distinguished boundary of $\Delta_{g}$ is homologous to a sum of local cycles separating local divisors $D_{j}=f_{j}=0, j=1, \ldots, s$, i.e. those cycles that are involved into definition of a local residue of Grothendieck. In the problem of representation of the integral (17) by a sum of local residues a principal role is played by the following notion.

Definition 1. A polyhedron $\Pi_{\mathrm{g}}$ is called compatible with a family of hypersurfaces (divisors) $\left\{D_{j}\right\}$, if the $j$ th facet of the polyhedron $\Pi_{\mathbf{g}}$ does not intersect $D_{j}$ for all $j, j=1, \ldots, s$.

Theorem 4 (The principle of separating cycles). If a polyhedron $\Pi_{\mathrm{g}}$ is bounded in compatible with the family of polar divisors $\left\{D_{j}\right\}$, then the integral (17) is equal to a sum of Grothendieck residues in $\Pi_{\mathbf{g}}$.

In case of an unbounded polyhedron, an additional condition of rapid decrease of the integrand in $\Pi_{\mathbf{g}}$ is required, similar to that in the classical Jordan lemma where instead of $\Pi_{\mathrm{g}}$ we have a half-plane. Such a condition is given in [8] and [7].

In the integral (16) the vertical subspace $\gamma+i \mathbb{R}^{2}$ can be seen as a distinguished boundary of some polyhedron. Note that in the case $N>1$ the number of such polyhedra is infinite. Our task is to divide polar hypersurfaces in (16) into 2 divisors and attach to $\gamma+i \mathbb{R}^{2}$ a polyhedron compatible with the obtained family. 
As a polyhedron we take

$$
\Pi=\left\{\operatorname{Re} u_{1}<\gamma_{1}, \operatorname{Re} u_{2}<\gamma_{2}\right\}
$$

The family of divisors we organize as follows: polar sets of Gamma functions $\Gamma\left(u_{1}\right)$ and $\Gamma\left(\frac{1}{4}\left(\mu_{2}+u_{1}-2 u_{2}\right)\right)$ (red and violet) we put into one divisor, while polar sets of $\Gamma\left(u_{2}\right)$ and $\Gamma\left(\frac{1}{4}\left(\mu_{1}-2 u_{1}+u_{2}\right)\right)$ (blue and green) into another. By gray lines we depict zeroes of the integrand (singularities of Gamma functions in the denominator and zeroes of $Q(u)$ ) (Fig. 1).

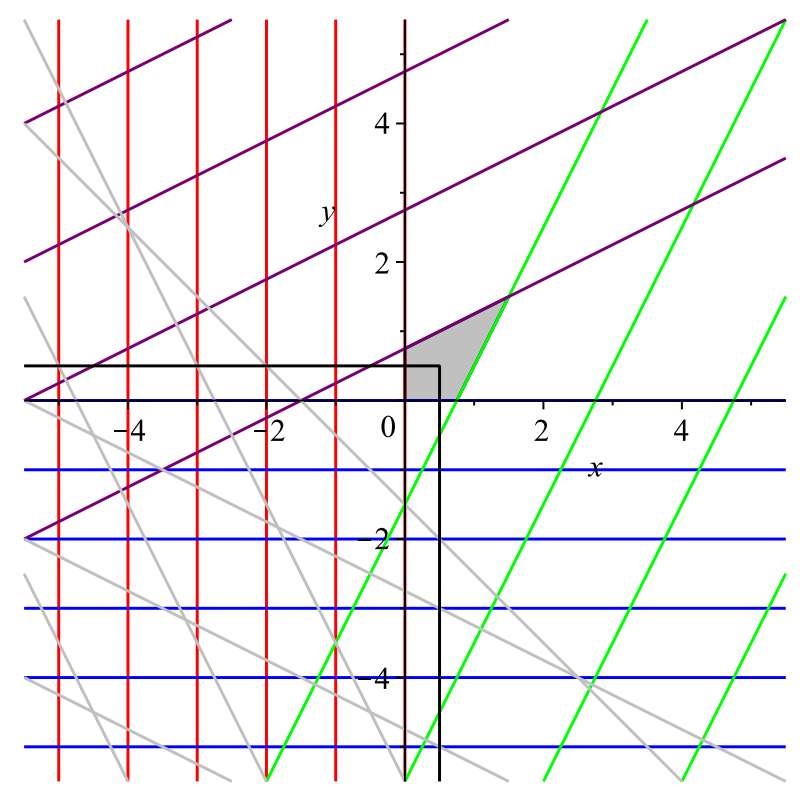

Fig. 1. Families of polar divisors of the integrand

It is easy to see that at the intersection points of oblique polar sets with vertical and horizontal ones inside the polyhedron the residue is 0 . Thus, in this case the integral (16) is a sum of residues over all points of the lattice $\mathbb{Z}_{\leqslant 0}^{2}$ where the integrand has a pole of the first order.

A pole at a point of $\mathbb{Z}_{\leqslant 0}^{2}$ gives an expression $c_{\mathbf{k}} \mathbf{x}^{\mathbf{k}}$, where $c_{\mathbf{k}}$ is defined in Theorem 3 by the formula (10). Summing up over all points of $\mathbb{Z}_{\leqslant 0}$ we obtain the series from Theorem 3 .

Thus, we have shown that the integral (3) under hypothesis of Theorem 1 has a non-empty convergence domain and represents a monomial of a solution of the system of algebraic equations (1). Moreover, computations show that the integral (16) coincides with the solution in the form of a hypergeometric series.

The research is carried out with the financial support of the RFBR, project no. 18-31-00193.

\section{References}

[1] L.Nilsson, Amoebas, Discriminants, and Hypergeometric Functions, Doctoral Thesis, Stockholm University, Sweden, 2009.

[2] I.A.Antipova, Siberian Math. J., 44(2003), no. 5, 757-764. DOI: 10.1023/A:1025920316227

[3] I.A.Antipova, Sb. Math., 198(2007), no. 4, 447-463. DOI: 10.4213/sm1562 
[4] V.A.Stepanenko, Vestn. KrasGU, 1(2003), 35-48 (in Russian).

[5] T.M.Sadykov, A.K.Tsikh, Hypergeometric and Algebraic Functions in Several Variables, Nauka, Moscow, 2014 (in Russian).

[6] V.V.Prasolov, Problems and Theorems in Linear Algebra, Amer. Math. Soc., Providence, 1994.

[7] O.N.Zhdanov, A.K.Tsikh, Siberian Math. J., 39(1998), no. 2, 245-260.

DOI: $10.1007 / \mathrm{BF} 02677509$

[8] M.Passare, A.Tsikh, O.Zhdanov, A multidimentional Jordan residue lemma with an application to Mellin-Barnes integrals, Contributions to complex analysis and analytic geometry. Braunschweig: Vieweg, 1994. P. 233-241. (Aspects Math. E; V.26). 47(2006), no. 2, 365-371.

[9] V.R.Kulikov, Siberian Math. J., 58(2017), no. 3, 493-499. DOI: 10.1134/S0037446617030120

[10] V.R.Kulikov, V.A.Stepanenko, St. Petersburg Math. J., 26(2015), no. 5, 839-848. DOI: $10.1090 / \mathrm{spmj} / 1361$

[11] I.Antipova, E.Kleshkova, V.Kulikov, Analytic continuation for solutions to the system of trinomial algebraic equations, 2019, arXiv:1905.01033v1 [math.CV].

\section{Гипергеометрические ряды и интегралы Меллина-Барнса для нулей системы полиномов Лорана}

Владимир Р. Куликов

Сибирский федеральный университет Красноярск, Российская Федерация

\footnotetext{
Аннотация. В работе приведен критерий сходимости интеграла Меллина-Барнса, представляющего нули системы полиномов Лорана. Представлена формула в виде кратного ряда гипергеометрического типа.
}

Ключевые слова: интегралы Меллина-Барнса, гипергеометрические ряды, полиномы Лорана. 